\title{
Cross-generational costs of compensatory growth in nine-spined sticklebacks
}

\begin{abstract}
Compensatory growth (CG) is a form of phenotypic plasticity allowing individuals' growth trajectories to rebound after a period of resource limitation, but little is known about the reproductive and cross-generational costs of CG. We studied the potential costs of CG by exposing female nine-spined sticklebacks Pungitius pungitius to 1) high (favourable), 2) low (stressful), and 3) recovery (initially stressful, subsequently favourable) feeding treatments, and quantified the effects of these treatments on female reproductive traits (clutch, egg and yolk size), and on the size of their offspring. The low feeding treatment reduced female size relative to the high and recovery feeding treatments, which produced equally large females. Hence, females from the recovery treatment demonstrated CG and full growth compensation. Feeding treatments had significant effects on clutch, yolk, egg and larval size, also when the effect of female size was controlled for. However, these effects came about mostly because females from the low feeding treatment produced small clutches with large eggs (containing little yolk) and larvae, whereas females from the recovery feeding treatment produced as large clutches, eggs (with similar amounts of yolk) and larvae as females from the high feeding treatment. Yet, structural equation modelling revealed that while a direct effect of female size on offspring size was positive in the low and high feeding treatments, it was negative in the recovery feeding treatment, independently of egg and clutch size. This indicates a cross-generational tradeoff between female and offspring sizes in the recovery feeding treatment. Also the tradeoff between clutch and larval size was more pronounced in recovery than in low or high feeding treatments. Hence, apart from demonstrating that environmental influences experienced by females during their development have the potential to influence their size, fecundity and reproductive traits, the results also provide evidence for complex cross-generational costs of recovery growth.
\end{abstract}

Keyword: Compensatory growth; Cross-generational costs; Nine-spined sticklebacks; Recovery feeding treatments 